\title{
Correction to: Development of a simultaneous LC-MS/MS method to predict in vivo drug-drug interaction in mice
}

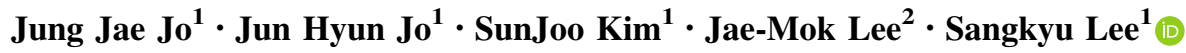

Published online: 13 November 2018

(C) The Pharmaceutical Society of Korea 2018

Correction to: Arch. Pharm. Res. (2018) 41:450-458

https://doi.org/10.1007/s12272-018-1012-8

The author would like to change conflict of interest statement of the online published article. The correct conflict of interest statement should read as:

\section{Conflict of interest}

The authors declare no conflict of interest.

The original article can be found online at https:// doi.org/10.1007/s12272-018-1012-8.

Jae-Mok Lee

leejm@knu.ac.kr

$\bowtie$ Sangkyu Lee

sangkyu@knu.ac.kr

1 BK21 Plus KNU Multi-Omics Based Creative Drug Research

Team, College of Pharmacy and Research Institute of

Pharmaceutical Sciences, Kyungpook National University,

Daegu 41566, Republic of Korea

2 Department of Periodontology, School of Dentistry, Kyungpook National University, 2177 Dalgubeol-daero, Jung-gu, Daegu 41940, Republic of Korea 